\title{
ABCB1 C3435T polymorphism influences the risk for Alzheimer's disease
}

\author{
Ágnes Fehér, Anna Juhász, Magdolna Pákáski, János Kálmán, Zoltán Janka \\ Department of Psychiatry, University of Szeged, 57 Kálvária Ave, Szeged H-6724, Hungary
}

\begin{abstract}
To evaluate the association of ATP binding cassette subfamily-B member-1 ( $A B C B 1)$ genetic variants with the susceptibility to Alzheimer's disease (AD), we genotyped the rs1128503 (C1236T), rs2032582 (G2677T/A) and rs1045642 (C3435T) polymorphisms in a case-control sample (234 AD patients, 225 controls). Single-marker analyses revealed significant association solely for rs1045642 polymorphism (C/C genotype carriers had increased risk for AD), which remains significant after correction for multiple testing. Haplotype analyses indicated three nominally significant associations which were lost after applying multiple test correction.
\end{abstract}




\section{Background}

Late-onset Alzheimer's disease (AD), the most common cause of dementia, is a complex neurodegenerative disorder with a strong genetic predisposition; however, in spite of the extensive research, the larger part of its heritable background is still an open question. At the molecular level, accumulation and aggregation of the amyloid- $\beta$ (A $\beta)$ peptides, leading to senile plaque formation; and hyperphosphorylation of the tau protein, resulting in neurofibrillary tangle generation, are the major features of AD pathology.

Adenosine-triphosphate (ATP) binding cassette subfamily B member 1 gene $(A B C B 1$; MIM\# 171050) at locus 7q21.1 encodes P-glycoprotein (P-gp), an efflux transporter that is located at the luminal side of the cerebral endothelial cells at the blood brain barrier (Pahnke et al., 2008). P-gp effectively prevents a number of structurally different drugs and toxicants from entering the brain and removes metabolic waste products from the brain, hence P-gp has an essential role in neuroprotection and overall brain homeostasis (Wolf et al., 2012). With respect to $\mathrm{AD}$, the involvement of $\mathrm{P}$-gp in $\mathrm{A} \beta$ transport is particularly important. Compared to healthy controls, $\mathrm{A} \beta$ clearance from the brain is significantly decreased in $\mathrm{AD}$ patients (Mawuenyega et al., 2010).

In vitro experiments provided evidence that $\mathrm{P}$-gp is capable of transporting $\mathrm{A} \beta$ (Lam et al., 2001; Kuhnke et al., 2007), and consistent with these findings, reduced P-gp activity via inhibition or genetic modification was reported to reduce $A \beta$ clearance from the brain in an AD mouse model (Cirrito et al., 2005). Additionally, the link between P-gp and AD is also supported by results on the inverse correlation between cerebral $\mathrm{A} \beta$ deposition and vascular P-gp expression at the blood brain barrier (Vogelgesang et al., 2002; Jeynes and Provias, 2011). 
The $A B C B 1$ gene is highly polymorphic. The rs1128503 single nucleotide polymorphism (SNP) is a nucleotide change in exon $12(\mathrm{C} 1236 \mathrm{~T})$ that does not alter the Glycine at position 412. The tri-allelic rs2032582 polymorphism in exon 21 (G2677T/A) leads to an Alanine to Threonine or Serine amino acid substitution (Ala893Thr/Ser). The rs1045642 synonymous polymorphism in exon $26(\mathrm{C} 3435 \mathrm{~T})$ does not affect the Ileucine at position 1145. To evaluate the possible association of $A B C B 1$ genetic variations with the susceptibility to late-onset AD, we performed a case-control study of a Hungarian sample genotyping the rs1128503, rs2032582 and rs1045642 polymorphisms.

\section{Subjects and methods}

A total of 234 Hungarian patients with late-onset $\mathrm{AD}$ (age: $75.6 \pm 6.8$ years (mean $\pm \mathrm{SD}$ ), men: $32.1 \%$ ) and 225 Hungarian, elderly, cognitively intact, healthy controls (age: $74.8 \pm 7.2$ years (mean $\pm \mathrm{SD}$ ), men: $33.3 \%$ ) were involved in the study. The AD patients were recruited from the Memory Clinic of the Department of Psychiatry, University of Szeged. A consensus clinical diagnosis of probable late-onset $\mathrm{AD}$ was established according to the National Institute of Neurological and Communicative Disorders and Stroke/Alzheimer's Disease and Related Disorders Association (NINCDS/ADRDA) criteria (McKhann et al., 1984). The minimum age at onset was 65 years. Global cognitive performance was measured by the Mini-Mental State Examination (MMSE). The mean MMSE score in the AD group was 17.3 \pm 5.7 (mean $\pm \mathrm{SD}$ ), while in the control group MMSE scores were higher than 28 points and none of the control probands had any verified symptoms of dementia. All recruitment and protocols were conducted with written informed consent and with the explicit approval of the Ethics Committee of the Hungarian Council on Science and Health (ETT-TUKEB). 
Genomic DNA was extracted from peripheral blood leukocytes using standard procedures. The investigated polymorphisms were genotyped using the method of PCR and enzyme digestion (PCR-RFLP). $A B C B 1$ rs1128503 genotypes were determined by a formerly described procedure with the restriction enzyme BsuRI (Sipeky et al., 2011). Genotyping of the $A B C B 1$ rs2032582 polymorphism was carried out as previously described with the restriction enzymes $B s e Y \mathrm{I}$ and $R s a \mathrm{I}$ (Cascorbi et al., 2004). Genotyping of the $A B C B 1$ rs 1045642 polymorphism was assessed by the same method described by Cascorbi et al. (2004) with the restriction enzyme MboI.

Genotype and allele frequencies were compared between patients and controls using Pearson's chi square and Fisher's exact tests. To exclude Type I errors, Bonferroni's correction for multiple testing was applied for 3 single-marker and 8 haplotype comparisons. The gender distribution of the $\mathrm{AD}$ and control groups was compared with Fisher's exact and the mean age was compared by using the t-test for independent samples. No statistically significant difference was found in the distribution of genders or in mean age between $A D$ and control groups $(p>0.05)$. Hardy-Weinberg Equilibrium (HWE) testing, linkage disequilibrium (LD) calculations and haplotype analyses were conducted using Haploview 4.2 (Barrett et al., 2005). Power analysis was performed using GPower 3.0 software (Faul et al., 2007) and the effect size was determined according to the method published by Cohen, 1988. Our study sample $(\mathrm{n}=459)$ has $81 \%$ power at the significance level of 0.05 to detect differences in rs1045642 genotypes between $\mathrm{AD}$ patients and controls (effect size: $\mathrm{w}=0.146$ ). 


\section{Results}

Genotype frequencies of the investigated polymorphisms in the $\mathrm{AD}$ and control groups applied to HWE $(p>0.05)$. LD and haplotype data were inferred from genotype data only for subjects not carrying the rs2032582 A allele because of its very low occurrence in both AD and control groups. Table 1 presents the LD characteristics of the genotyped polymorphisms. Moderate LD was observed between rs1128503 and rs2032582 ( $\left.{ }^{\prime}=0.749, r^{2}=0.434\right)$, and between rs2032582 and rs1045642 polymorphisms $\left(D^{\prime}=0.729, r^{2}=0.376\right)$, and weak LD was detected between rs1128503 and rs1045642 polymorphisms ( $\left.\mathrm{D}^{\prime}=0.474, \mathrm{r}^{2}=0.203\right)$.

The genotype and haplotype frequencies of the $A B C B 1$ polymorphisms are summarized in Table 2. The ratio of the different rs1128503 genotypes was similar in the AD and in the control groups, and showed no statistically significant difference $\left(\chi^{2}=0.506\right.$ (2) $p=0.776$ ). Regarding the rs 2032582 polymorphism, no A/A genotype carriers were found, besides, the G/A and the T/A genotypes had a very low frequency in both investigated groups. The $\mathrm{G} / \mathrm{G}$ and $\mathrm{G} / \mathrm{T}$ genotypes occurred at a slightly higher frequency in the $\mathrm{AD}$ than in the control group; however, the difference was not statistically significant $\left(\chi^{2}=3.951\right.$ (4) $\left.p=0.413\right)$. Compared with the controls, the rs $1045642 \mathrm{C} / \mathrm{C}$ genotype was significantly over-represented in the $\mathrm{AD}$ group $\left(\chi^{2}=9.840\right.$ (2) $p=0.007$, corrected: $\left.p=0.021\right)$. The $\mathrm{C} / \mathrm{C}$ genotype carriers had a significantly increased risk for $\mathrm{AD}(\mathrm{OR}=2.29,95 \% \mathrm{CI}: 1.35-3.88, p=0.002)$ considering the $\mathrm{T} / \mathrm{T}$ genotype carriers as reference category.

The predominant haplotypes of the markers rs1128503, rs2032582 and rs1045642 were the C-G-C and T-T-T in both AD and control groups. Our haplotype analyses revealed three nominally significant associations (Table 2). The T-T-T and T-T-C haplotypes were more frequent in the control group than in the $\mathrm{AD}$ group (T-T-T: $p=0.040$; corrected $p=0.320$; 
T-T-C: $p=0.016$; corrected $p=0.128$ ), while the C-T-T haplotype had a higher occurrence in the $\mathrm{AD}$ group ( $p=0.032$; corrected $p=0.256)$.

\section{Discussion}

In single-marker case-control analyses, we found a significant association only for rs 1045642 polymorphism out of the three investigated SNPs. Even after correcting $p$ values using the Bonferroni's method for multiple tests, this significant correlation was still found. Significantly increased susceptibility to AD associated with rs1045642 C/C genotype was found considering $\mathrm{T} / \mathrm{T}$ genotype as the reference category. The genotype distributions of the rs1128503 and rs2032582 polymorphisms were similar in the AD and control groups without a statistically significant difference.

Even though the synonymous rs1045642 polymorphism does not produce altered coding sequence, it seems to have functional consequences, since it was reported to result in lower intestinal P-gp expression and increased oral bioavailability of the Pgp substrate digoxin, decreased mRNA stability and expression, and reduced protein function (Hoffmeyer et al., 2000; Wang et al., 2005; Assema et al., 2012). On the other hand, reports of conflicting results can also be found (Sakaeda et al., 2001; Nakamura et al., 2002).

The frequencies of the investigated $A B C B 1$ genotypes in our control sample are comparable to earlier reports on other control populations of Caucasian origin (Cascorbi et al., 2004; Sipeky et al., 2011). Two case-control studies with small and medium sample size reported no association between rs2032582 or rs1045642 polymorphism and the risk for AD (Frankfort et al., 2006; Kohen et al., 2011), and two genome wide association studies also did not detect correlation between rs 1045642 polymorphism and the genetic susceptibility to AD 
(Reiman et al., 2007; Li et al., 2008). The ABCB1 polymorphisms were also studied in a histopathologically confirmed $\mathrm{AD}$ case-control sample and no effect of $\mathrm{ABCB} 1$ variants was found in the entire cohort; however, a significant association was detected between rs 2032582 genotypes and $\mathrm{AD}$ risk among females and individuals older than 65 years (Cascorbi et al., 2013).

Frankfort and co-workers showed evidence for the correlation between ABCB1 rs1128503 and rs2032582 genotypes and $A \beta$ serum profile, and therefore suggested further research on the potential involvement of $\mathrm{ABCB} 1$ genetic variations in $\mathrm{AD}$ (Frankfort et al., 2008). Another investigation revealed significant differences in peripheral leukocyte gene expression profiles between $\mathrm{AD}$ patients and non-demented controls, and found that $\mathrm{ABCB} 1$ gene had decreased expression in $\mathrm{AD}$ and the $\mathrm{ABCB} 1$ expression levels were positively correlated with MMSE score (Chen et al., 2011).

The investigated polymorphisms are not strictly allelic, they showed LD, hence we also performed the haplotype estimation between cases and controls, and found moderate (between rs1128503 and rs2032582, and between rs2032582 and rs1045642 polymorphisms) and weak LD (between rs1 128503 and rs1045642 polymorphisms) in our sample. Consistent with findings of previous studies, the $\mathrm{C}-\mathrm{G}-\mathrm{C}$ and $\mathrm{T}-\mathrm{T}-\mathrm{T}$ were the predominant haplotypes of the rs1128503, rs2032582, and rs1045642 polymorphisms in both investigated groups. Haplotype analyses revealed three nominally significant associations for T-T-T, T-T-C and CT-T haplotypes, however, they did not remain significant after applying the Bonferroni's correction for multiple tests.

The present study led to the conclusion that $A B C B 1$ rs1045642 polymorphism may have a role in the genetic risk for developing $\mathrm{AD}$. Subjects carrying the $\mathrm{C} / \mathrm{C}$ genotype of ABCB1 gene appeared to exhibit AD significantly more frequently. The medium size of our 
sample, an important limitation, should be mentioned, and further investigations with considerably larger samples and in detail using more markers are required.

\section{Acknowledgments}

The authors are grateful to the participants of this study for their cooperation. This work was supported by a grant from TÁMOP-4.2.2A-11/1/KONV-2012-0052. 


\section{References}

1. Barrett J.C., Fry B., Maller J. and Daly M.J. (2005) Haploview: analysis and visualization of LD and haplotype maps. Bioinformatics. 21, 263-265.

2. Cascorbi I., Flüh C., Remmler C., et al. (2013) Association of ATP-binding cassette transporter variants with the risk of Alzheimer's disease. Pharmacogenomics. 14, 485-494.

3. Cascorbi I., Gerloff T., Johne A., et al. (2004) Frequency of single nucleotide polymorphisms in the P-glycoprotein drug transporter MDR1 gene in white subjects. Clin. Pharmacol. Ther. 69, 169-174.

4. Chen K.D., Chang P.T., Ping Y.H., Lee H.C., Yeh C.W. and Wang P.N. (2011) Gene expression profiling of peripheral blood leukocytes identifies and validates $\mathrm{ABCB} 1$ as a novel biomarker for Alzheimer's disease. Neurobiol Dis. 43, 698-705.

5. Cirrito JR, Deane R, Fagan AM, et al. (2005) P-glycoprotein deficiency at the blood-brain barrier increases amyloid-beta deposition in an Alzheimer's disease mouse model. J. Clin. Invest. 115, 3285-3290.

6. Cohen J. (1988) Statistical power analysis for the behavioral sciences, second ed., Lawrence Erlbaum Associates Inc., Hillsdale, New Jersey, pp. 216-226.

7. Faul F., Erdfelder E., Lang A.G. and Buchner A. (2007) G*Power 3: A flexible statistical power analysis program for the social, behavioral, and biomedical sciences. Behavior Research Methods. 39, 175-191.

8. Frankfort S.V., Doodeman V.D., Bakker R., et al. (2006) ABCB1 genotypes and haplotypes in patients with dementia and age-matched non-demented control patients. Mol. Neurodegener. 1, 13.

9. Frankfort S.V., van Campen J.P., Tulner L.R. and Beijnen J.H. (2008) Serum amyloid beta peptides in patients with dementia and age-matched non-demented controls as 
detected by surface-enhanced laser desorption ionisation-time of flight mass spectrometry (SELDI-TOF MS). Curr Clin Pharmacol. 3, 144-154.

10. Hoffmeyer S., Burk O., von Richter O., et al. (2000) Functional polymorphisms of the human multidrug-resistance gene: multiple sequence variations and correlation of one allele with P-glycoprotein expression and activity in vivo. Proc Natl Acad Sci USA. 97, 3473-3478.

11. Jeynes B. and Provias J. (2011) An investigation into the role of P-glycoprotein in Alzheimer's disease lesion pathogenesis. Neurosci. Lett. 487, 389-393.

12. Kohen R., Shofer J.B., Korvatska O., et al. (2011) ABCB1 genotype and CSF betaamyloid in Alzheimer disease. J. Geriatr. Psychiatry. Neurol. 24, 63-66.

13. Kuhnke D., Jedlitschky G., Grube M., et al. (2007) MDR1- P-glycoprotein (ABCB1) mediates transport of Alzheimer's amyloid-beta peptides - implications for the mechanisms of beta-amyloid clearance at the blood-brain barrier. Brain Pathol. 17, 347353.

14. Lam F.C., Liu R., Lu P., et al. (2001) Beta-amyloid efflux mediated by p-glycoprotein. J. Neurochem. 76, 1121-1128.

15. Li H., Wetten S., Li L., et al. (2008) Candidate single-nucleotide polymorphisms from a genomewide association study of Alzheimer disease. Arch. Neurol. 65, 45-53.

16. Mawuenyega K.G., Sigurdson W., Ovod V., et al. (2010) Decreased clearance of CNS beta-amyloid in Alzheimer's disease. Science. 330, 1774.

17. McKhann G., Drachman D., Folstein M., Katzman R., Price D. and Stadlan E.M. (1984) Clinical diagnosis of Alzheimer's disease: report of the NINCDS-ADRDA Work Group under the auspices of Department of Health and Human Services Task Force on Alzheimer's Disease. Neurology. 34, 939-944. 
18. Nakamura T., Sakaeda T., Horinouchi M., et al. (2002) Effect of the mutation (C3435T) at exon 26 of the MDR1 gene on expression level of MDR1 messenger ribonucleic acid in duodenal enterocytes of healthy Japanese subjects. Clin Pharmacol Ther. 71, 297-303.

19. Pahnke J., Wolkenhauer O., Krohn M.A. and Walker L.C. (2008) Clinico-pathologic function of cerebral $\mathrm{ABC}$ transporters - implications for the pathogenesis of Alzheimer's disease. Curr. Alzheimer Res. 5, 396-405.

20. Reiman E.M., Webster J.A., Myers A.J., et al. (2007) GAB2 alleles modify Alzheimer's risk in APOE epsilon4 carriers. Neuron. 54, 713-720.

21. Sakaeda T., Nakamura T., Horinouchi M., et al. (2001) MDR1 genotype-related pharmacokinetics of digoxin after single oral administration in healthy Japanese subjects. Pharm Res. 18, 1400-1404.

22. Sipeky C., Csongei V., Jaromi L., et al. (2011) Genetic variability and haplotype profile of MDR1 (ABCB1) in Roma and Hungarian population samples with a review of the literature. Drug Metab. Pharmacokinet. 26, 206-215.

23. van Assema D.M.E., Lubberink M., Rizzu P., et al. (2012) Blood-brain barrier Pglycoprotein function in healthy subjects and Alzheimer's disease patients: effect of polymorphisms in the $\mathrm{ABCB} 1$ gene.

24. Vogelgesang S., Cascorbi I., Schroeder E., et al. (2002) Deposition of Alzheimer's betaamyloid is inversely correlated with P-glycoprotein expression in the brains of elderly non-demented humans. Pharmacogenetics. 12, 535-541.

25. Wang D., Johnson A.D., Papp A.C., Kroetz D.L. and Sadee W. (2005) Multidrug resistance polypeptide 1 (MDR1, ABCB1) variant 3435C $>\mathrm{T}$ affects mRNA stability. Pharmacogenet Genomics. 15, 693-704.

26. Wolf A., Bauer B. and Hartz A.M. (2012) ABC Transporters and the Alzheimer's disease Enigma. Front. Psychiatry. 3, 54. 
Table 1. Linkage disequilibrium characteristics of the investigated $A B C B 1$ polymorphisms

\begin{tabular}{llllllll}
\hline \multicolumn{2}{l}{ Polymorphisms } & \multicolumn{2}{c}{ MAF (\%) } & $p$ value & \multicolumn{2}{c}{ Linkage disequilibrium } \\
& & AD patients & Controls & & with & $D^{\prime}$ & $\mathrm{r}^{2}$ \\
\hline rs1128503 & $1236 \mathrm{C}>\mathrm{T}$ & $48.3 \%$ & $49.6 \%$ & 0.702 & rs2032582 & 0.749 & 0.434 \\
rs2032582 & $2677 \mathrm{G}>\mathrm{T}$ & $41.2 \%$ & $45.8 \%$ & 0.170 & rs1045642 & 0.729 & 0.376 \\
rs1045642 & $3435 \mathrm{C}>\mathrm{T}$ & $46.4 \%$ & $56.7 \%$ & $0.002 * *$ & rs1128503 & 0.474 & 0.203 \\
\hline
\end{tabular}

ABCB 1: ATP binding cassette subfamily B member 1; MAF: minor allele frequency; AD: Alzheimer's disease "Linkage disequilibrium data were inferred from genotype data only for subjects not carrying the A allele of the rs2032582 polymorphism.

Table 2. Genotype and haplotype frequencies of the investigated $A B C B 1$ polymorphisms

\begin{tabular}{|c|c|c|c|c|}
\hline & AD patients & Controls & chi square & $p$ value \\
\hline $\begin{array}{l}\text { rs1128503 } \\
\mathrm{C} / \mathrm{C} \\
\mathrm{C} / \mathrm{T} \\
\mathrm{T} / \mathrm{T}\end{array}$ & $\begin{array}{l}56(23.9 \%) \\
130(55.6 \%) \\
48(20.5 \%)\end{array}$ & $\begin{array}{l}54(24.0 \%) \\
119(52.9 \%) \\
52(23.1 \%)\end{array}$ & 0.506 & 0.776 \\
\hline $\begin{array}{l}\text { rs2032582 } \\
\text { G/G } \\
\text { G/T } \\
\text { T/T } \\
\text { G/A } \\
\text { T/A } \\
\text { A/A } \\
\end{array}$ & $\begin{array}{l}69(29.5 \%) \\
123(52.6 \%) \\
30(12.8 \%) \\
9(3.8 \%) \\
3(1.3 \%) \\
\text { ND }\end{array}$ & $\begin{array}{l}62(27.6 \%) \\
109(48.4 \%) \\
44(19.6 \%) \\
8(3.5 \%) \\
2(0.9 \%) \\
\text { ND }\end{array}$ & 3.951 & 0.413 \\
\hline $\begin{array}{c}\text { rs1045642 } \\
\mathrm{C} / \mathrm{C} \\
\mathrm{C} / \mathrm{T} \\
\mathrm{T} / \mathrm{T}\end{array}$ & $\begin{array}{l}66(28.2 \%) \\
119(50.9 \%) \\
49(20.9 \%)\end{array}$ & $\begin{array}{l}43(19.1 \%) \\
109(48.4 \%) \\
73(32.5 \%)\end{array}$ & 9.840 & $0.007 * *$ \\
\hline $\begin{array}{c}\text { Haplotypes }^{\#} \\
\text { C-G-C } \\
\text { T-T-T } \\
\text { C-G-T } \\
\text { T-G-C } \\
\text { T-T-C } \\
\text { C-T-T } \\
\text { T-G-T } \\
\text { C-T-C }\end{array}$ & $\begin{array}{l}172(36.9 \%) \\
141(30.1 \%) \\
46(9.8 \%) \\
41(8.8 \%) \\
25(5.4 \%) \\
12(2.5 \%) \\
19(4.0 \%) \\
12(2.5 \%)\end{array}$ & $\begin{array}{l}146(32.4 \%) \\
164(36.5 \%) \\
54(11.9 \%) \\
35(7.7 \%) \\
10(2.3 \%) \\
23(5.2 \%) \\
14(3.1 \%) \\
4(0.9 \%)\end{array}$ & $\begin{array}{l}2.008 \\
4.227 \\
1.070 \\
0.384 \\
5.780 \\
4.582 \\
0.575 \\
3.625 \\
\end{array}$ & $\begin{array}{l}0.157 \\
0.040^{*} \\
0.301 \\
0.536 \\
0.016^{*} \\
0.032^{*} \\
0.448 \\
0.057\end{array}$ \\
\hline
\end{tabular}

$A B C B$ 1: ATP binding cassette subfamily B member 1; AD: Alzheimer's disease; ND: not detected

${ }^{\#}$ Haplotypes of the rs1128503, rs2032582 and rs1045642 polymorphisms. Haplotype data were inferred from genotype data only for subjects not carrying the A allele of the rs2032582 polymorphism. Chi squares and $p$ values for comparisons of the haplotype frequencies were determined by using the Haploview 4.2 program. 